\title{
Vulvar Crohn disease
}

\author{
Riki Dayan MD, Leslie Sadownik MD MEd, Jason Reutter MD
}

Cite as: CMAJ 2021 March 15;193:E379. doi: 10.1503/cmaj.190112

A 35-year-old woman with a 15-year history of gastrointestinal Crohn disease presented to a multidisciplinary vulvar clinic with an 18-month history of symptomatic vulvar bumps. She reported intermittent vulvar pain and swelling. A superficial skin biopsy performed 2 years earlier by her family physician had shown only mild keratosis. The lesions had been treated unsuccessfully with trichloroacetic acid for presumed condylomata. Findings on clinical examination included bilateral labial edema, thickening of the labia majora and minora and flesh-coloured papules (Figure 1A). These findings were suggestive of acquired lymphangectasia from vulvar Crohn disease, supported by the presence of noncaseating granulomas on biopsy (Figure 1B). The lesions responded well to $\mathrm{CO}_{2}$ laser vapourization. Since the patient had also been recently started on vedolizumab for her gastrointestinal Crohn disease, this may eventually improve the vulvar edema.

Vulvar Crohn disease is uncommon, but it can adversely affect quality of life, and is often under-recognized and misdiagnosed. ${ }^{1-3}$ The median time to diagnosis of vulvar Crohn disease is 3.5 years, as patients often see multiple specialists before a diagnosis is established. ${ }^{2}$ Although most patients (50\%-80\%) with this condition have underlying gastrointestinal Crohn disease, not all patients with vulvar Crohn disease will go on to develop bowel disease. ${ }^{1,2}$

Nonspecific associated gynecologic symptoms, such as itch, make the diagnosis challenging. ${ }^{2}$ Vulvar findings include edema, labial hypertrophy, lymphangiectasia, knife-like fissures, ulcerations, abscesses and fistulas..$^{1-3}$ Histological findings, such as the presence of noncaseating granulomas, granulomatous vasculitis or lymphangitis in the dermis, support the diagnosis. ${ }^{1-3}$ Other diagnoses to consider in presentations similar to our patient's include skin tags, condylomata, molluscum contagiosum, vulvar intraepithelial neoplasia and acquired lymphagiectasia. ${ }^{4}$

Vulvar Crohn disease is a chronic disease that involves long-term medical (e.g., antibiotics, systemic corticosteroids or anti-tumour necrosis factor- $\alpha$ therapies) or surgical ( $\mathrm{CO}_{2}$ laser ablation, excision) management. ${ }^{2}$ It may be challenging to manage and it benefits from a multidisciplinary approach, involving gastroenterologists, gynecologists, pathologists and dermatologists..$^{1,2}$ In patients with known Crohn disease and abnormal vulvar findings, the possibility of underlying vulvar Crohn disease should be considered. ${ }^{3}$

\section{References}

1. Barret M, de Parades V, Battistella M, et al. Crohn's disease of the vulva. J Crohns Colitis 2014;8:563-70.

2. Boxhoorn L, Stoof TJ, de Meij T, et al. H. Clinical experience and diagnostic algorithm of vulval Crohn's disease. Eur J Gastroenterol Hepatol 2017;29:838-43.

3. Feller ER, Ribaudo S, Jackson ND. Gynecologic aspects of Crohn's disease. Am Fam Physician 2001;64:1725-8.

4. Stewart CJR, Chan T, Platten M. Acquired lymphangiectasi ('lymphangioma circumscriptum') of the vulva: a report of eight cases. Pathology 2009;41:448-53.

\section{Competing interests: None declared.}

This article has been peer reviewed.

The authors have obtained patient consent.

Affiliations: Department of Obstetrics and Gynecology (Dayan), Faculty of Medicine, The University of British Columbia; BC Centre for Vulvar Health (Sadownik), Gordon and Leslie Diamond Health Care Centre; Departments of Gynecology, and Dermatology and Skin Science (Sadownik), The University of British Columbia; BC Centre for Vulvar Health (Sadownik); Vancouver Coastal Health Authority (Sadownik), Vancouver, BC; Dermatopathology (Reutter), Piedmont Pathology Associates Inc., Hickory, NC.

Content licence: This is an Open Access article distributed in accordance with the terms of the Creative Commons Attribution (CC BYNC-ND 4.0) licence, which permits use, distribution and reproduction in any medium, provided that the original publication is properly cited, the use is noncommercial (i.e., research or educational use), and no modifications or adaptations are made. See: https:// creativecommons.org/licenses/by-nc-nd/4.0/

Correspondence to: Riki Dayan, r.dayan@alumni.ubc.ca 